\title{
Accuracy of Magnetic Resonance Imaging in Diagnosis of Internal Derangement of the Knee
}

\author{
Mohammad M. Abbas, MD, Abdullah A. Abulaban 1 , MBBS \\ and Mamdooh M. Kotb' ${ }^{2}$, MD, PhD
}

Department of Orthopedics, 'House Officer, and ${ }^{2}$ Department of Diagnostic Radiology

Faculty of Medicine, King Abdulaziz University, Jeddah, Saudi Arabia

\section{Correspondence}

Dr. Mohammad M. Abbas

P.O. Box 80215, Jeddah 21589, Saudi Arabia

e.M: mmabbas@kau.edu.sa

Submission: 16 Jul. 2016

Accepted: 25 Aug. 2016

\section{Citation}

Abbas MM, Abulaban AA, Kotb MM. Accuracy of magnetic resonance imaging in diagnosis of internal derangement of the knee. JKAU Med Sci 2016; 23 (4): 11-17. DOl: 10.4197/Med. 23.4.2

\begin{abstract}
The current study aims to assess the sensitivity and specificity of magnetic resonance imaging by reference to arthroscopy in the preoperative diagnosis of knee pathologies. In this retrospective study, pre-operative knee magnetic resonance imaging reports of 70 patients were reviewed by a blinded musculoskeletal radiologist and compared with the respective arthroscopic findings recorded in the operative notes. Pre-operative magnetic resonance imaging demonstrated a sensitivity level of $92.7 \%$ and $61.3 \%$ and specificity level of $33.3 \%$ and $53.9 \%$ in detecting pathologies of the medial and lateral menisci, respectively. With respect to the cases of complete anterior cruciate ligament tear, sensitivity was $76.7 \%$ with $92.5 \%$ specificity. On the other hand, magnetic resonance imaging demonstrated $100 \%$ sensitivity and $90.7 \%$ specificity in the diagnosis of posterior cruciate ligament partial tear. Relatively better sensitivity in detecting cruciate ligaments pathology and better specificity in detecting menisci pathology was reported using magnetic resonance imaging. Almost $25 \%$ of complete anterior cruciate ligament injuries may remain undetected in preoperative magnetic resonance imaging reports, while they represent the most prevalent knee injury. Magnetic resonance imaging should be used with caution in knee pathology and arthroscopy continues to be the gold standard.
\end{abstract}

\section{Keywords}

Arthroscopy; Cruciate ligaments; Knee; Magnetic resonance imaging; Meniscus

\section{Introduction}

usculoskeletal (MSK) imaging and in particular magnetic resonance imaging (MRI) is an important diagnostic, teaching and research tool for health care providers ${ }^{[1]}$. In knee surgery, it has often been regarded as a promising noninvasive alternative to diagnostic arthroscopy. Thus, clinicians prefer performing MRI scan to support the diagnosis of knee pathology, prior to recommending arthroscopic examination and the surgery. A small randomized controlled trial in the National Health Service in England suggested that conducting an MRI-based diagnosis of knee disorders might curtail the need for performing 
arthroscopy. This could reduce arthroscopy rates, which in turn, improves the overall patient outcome ${ }^{[2]}$.

A systematic meta-analysis including 29 articles published between 1991 and 2000 reported that MRI is an appropriate screening tool for therapeutic arthroscopy; eliminating the need for diagnostic arthroscopy in most cases ${ }^{[3]}$.

Other observational non-randomized studies in the United Kingdom, Austria, and the United States also concluded that an MRI scan presents a more costeffective presurgical diagnostic tool, as compared to knee arthroscopy. Owing to this, almost $25 \%$ to $50 \%$ of patients on a waiting list for surgery avoided arthroscopy by the prior use of MRI ${ }^{[4]}$.

Madhusudhan et $a l^{[5]}$ and Ercin et al. ${ }^{[6]}$ found that using MRI as a routine supplement to the clinical examination is unnecessary, as thorough clinical assessment can allow the experienced surgeon to make a definite primary diagnosis. Moreover, based on a retrospective study of 288 knee arthroscopies in a community setting, Hardy et al. ${ }^{[7]}$ also concluded that in case of suspected meniscal pathology, MRI should be requested only when the orthopedic clinical diagnosis is ambiguous. Recently, a rampant trend has been observed in clinical practice to order MRI after clinical examination and radiographs in the diagnosis of potential knee soft tissue and articular cartilage abnormalities ${ }^{[8]}$.

Although arthroscopy is a highly sensitive and specific procedure with both diagnostic and therapeutic advantages, it is an invasive procedure and entails several potential intraoperative and postoperative complications ${ }^{[9,10]}$. Thus, the decision to perform an arthroscopy warrants the need of a riskbenefit analysis on a case-to-case basis. The current study was conceptualized to assess the diagnostic value of pre-operative MRI in internal knee derangement, and whether it can be reliably used to screen for or rule out knee lesions. Sensitivity, specificity positive predictive value (PPV), negative predictive value (NPV) and accuracy of pre-operative MRI were analyzed, by reference to arthroscopy in the diagnosis of different interior knee lesions, with focus on tears of the menisci and cruciate ligaments.

\section{Methods}

Ethical approval for the current study was obtained from the Biomedical Ethics Committee (No. 888-12) at King Abdulaziz University Hospital (KAUH), Jeddah, Saudi Arabia. Patients who had undergone an MRI examination of the knee in KAUH and subsequent arthroscopy, during an 8-year period from May 11, 2004 to March 11, 2012, were included in the study. A retrospective search of the hospital database was conducted, identifying 70 patients who fulfilled the inclusion criterion.

Magnetic resonance images were obtained with a dedicated knee coil at either 1.5 T (MAGNETOM Symphony Tim, Siemens Medical Systems, Erlangen, Germany) or with 3T MRI system (MAGNETOM Verio, Siemens Medical Systems, Erlangen, Germany) with a standard protocol.

After data collection, a blinded radiologist with interest in musculoskeletal radiology examined and analyzed the reports of all MRI scans that were performed prior to knee arthroscopy (average duration between the MRI and the arthroscopy was eight months). The criteria used to define a cruciate ligament tear were discontinuity, abnormal signal intensity, and indistinct margins of the ligament. The diagnostic criterion for meniscal tear was abnormal signal intensity within the meniscus that extended to the meniscal articular surface or abnormal morphology of the meniscus ${ }^{[11]}$.

Arthroscopic findings were extracted from the operative notes of the 70 patients by using the hospital information system.

In this study, meniscal tear was considered as one group and compared against the degenerative and the normal menisci. In addition, the complete anterior cruciate ligament $(\mathrm{ACL})$ tear was considered as one group compared against the normal and partially torn ACLs.

Data was entered and analyzed using the Statistical Package for Social Sciences version 16.0 for Windows (SPSS Inc., Chicago, IL, USA). Sensitivity, specificity, accuracy, positive predictive value (PPV) and negative predictive value (NPV) of MRI in the diagnosis of cruciate ligaments (anterior and posterior) and menisci (medial and lateral) tears were analyzed by comparison with knee arthroscopy results, the gold standard. Sensitivity and specificity were measured as the proportion of actual positive and negative findings that were correctly identified, respectively. Sensitivity was calculated as the number of true positives divided 
by the number of cases that had positive arthroscopy; and specificity as the number of true negatives divided by the number of cases that had negative arthroscopy. Positive predictive value (PPV) was calculated as the proportion of patients with positive test results (positive MRI) who were correctly diagnosed (positive arthroscopy); and negative predictive value (NPV) as the proportion of patients with negative test results (negative MRI) who were correctly diagnosed (negative arthroscopy). Accuracy was measured as the percentage of patients in whom MRI scans revealed correct findings, which included both true positive and true negative results.

\section{Results}

A total of 70 patients with mean (SD) age of 39.26(16.81) years, who were suspected to have either meniscus or ligament injury were included in the study.

\section{Anterior Cruciate Ligament (ACL) Tear}

There were $30(42.86 \%)$ cases diagnosed as complete $\mathrm{ACL}$ tear with arthroscopy, and no case of partial $\mathrm{ACL}$ tear. In MRI, 26 knees were classified as complete tear and 8 as partial tears. Consequently, MRI helped detecting $\mathrm{ACL}$ tear (partial or complete) with $86.7 \%$ (95\% Cl $=69.3 \%$; 96.2\%) sensitivity, 80.0\% (64.4; 91.0\%) specificity, 76.5\% (63.3; 86.0\%) PPV and 88.9\% (76.0;
95.3\%) NPV. Regarding detection of complete ACL tear (versus no complete tear), MRI was less sensitive and more specific; sensitivity $=76.7 \%(57.7 ; 90.1 \%)$; specificity $=92.5 \%(79.6 ; 98.4 \%)$ (Table 1).

\section{Posterior Cruciate Ligament (PCL) Tear}

Posterior cruciate ligament tear was diagnosed in 27 (38.57\%) cases, in arthroscopy; all being correctly detected as such in MRI, while 4 others were falsely detected as PCL tear. Consequently, MRI helped diagnosing $\mathrm{PCL}$ tear with $100 \%$ sensitivity (87.2; 100.0\%), 90.7\% (77.9; 98.4\%) specificity; 87.1\% (72.6; 94.5\%) PPV, and $100.0 \%$ NPV (Table 2).

\section{Medial Meniscal (MM) Tear}

Medial meniscal tear was diagnosed in arthroscopy in $55(78.57 \%)$ cases, of which 51 were correctly detected in MRI. Thus, MRI helped diagnosing MM tear with 92.7\% (82.41; 98.0\%) sensitivity, 33.3\% (11.8; 61.6\%) specificity, 83.6\% (78.0; 88.0\%) PPV, 55.6\% (27.7; 80.3\%) NPV, and $80.0 \%$ accuracy (Table 3 ).

\section{Lateral Meniscal (LM) Tear}

A total of $31(44.29 \%)$ patients had lateral meniscus (LM) tear, of which only 19 were correctly identified and 18 were falsely identified as LM tear in MRI. Thus, MRI

Table 1. Diagnostic value of magnetic resonance imaging in anterior cruciate ligament tear.

\begin{tabular}{|l|c|c|c|c|}
\hline \multirow{2}{*}{ Parameter } & \multicolumn{2}{|c|}{ Partial or Complete ACL Tear } & \multicolumn{2}{c|}{ Complete ACL Tear } \\
\cline { 2 - 5 } & Value & $95 \% \mathrm{Cl}$ & Value & 95\%Cl \\
\hline Sensitivity & $86.67 \%$ & $69.28 \%$ to $96.24 \%$ & $76.67 \%$ & $79.72 \%$ to $90.07 \%$ \\
\hline Specificity & $80.00 \%$ & $64.35 \%$ to $90.95 \%$ & $92.50 \%$ & $3.61 \%$ to $98.43 \%$ \\
\hline Positive Likelihood Ratio & 4.33 & 2.30 to 8.18 & 10.22 & 0.38 to 30.90 \\
\hline Negative Likelihood Ratio & 0.17 & 0.07 to 0.42 & 0.25 & - \\
\hline Accuracy & $82.85 \%$ & - & $85.71 \%$ & 71.72 to 0.49 \\
\hline Positive Predictive Value & $76.47 \%$ & $63.25 \%$ to $85.99 \%$ & $88.46 \%$ & $7.86 \%$ \\
\hline Negative Predictive Value & $88.89 \%$ & $76.02 \%$ to $95.28 \%$ & $84.09 \%$ & 73.31 to $91.05 \%$ \\
\hline
\end{tabular}

ACL: Anterior Cruciate Ligament; Cl: Confidence interval

Table 2. Diagnostic value of magnetic resonance imaging in posterior cruciate ligament tear.

\begin{tabular}{|l|c|c|}
\hline \multicolumn{1}{|c|}{ Parameter } & Value & $95 \% \mathrm{Cl}$ \\
\hline Sensitivity & $100.00 \%$ & $87.23 \%$ to $100.00 \%$ \\
\hline Specificity & $90.70 \%$ & $77.86 \%$ to $97.41 \%$ \\
\hline Positive Likelihood Ratio & 4.33 & 2.30 to 8.18 \\
\hline Negative Likelihood Ratio & 0.17 & 0.07 to 0.42 \\
\hline Accuracy & $89.9 \%$ & - \\
\hline Positive Predictive Value & $87.10 \%$ & $72.64 \%$ to $94.49 \%$ \\
\hline Negative Predictive Value & $100.00 \%$ & - \\
\hline Cl: Confidence interval
\end{tabular}


Table 3. Diagnostic value of magnetic resonance imaging in medical meniscus tear.

\begin{tabular}{|l|c|c|}
\hline \multicolumn{1}{|c|}{ Parameter } & Value & $95 \% \mathrm{Cl}$ \\
\hline Sensitivity & $92.73 \%$ & $82.41 \%$ to $97.98 \%$ \\
\hline Specificity & $33.33 \%$ & $11.82 \%$ to $61.62 \%$ \\
\hline Positive Likelihood Ratio & 1.39 & 0.97 to 2.00 \\
\hline Negative Likelihood Ratio & 0.22 & 0.07 to 0.71 \\
\hline Accuracy & $80.00 \%$ & - \\
\hline Positive Predictive Value & $83.61 \%$ & $77.97 \%$ to $88.02 \%$ \\
\hline Negative Predictive Value & $55.56 \%$ & $27.66 \%$ to $80.34 \%$ \\
\hline
\end{tabular}

cl: Confidence interval

Table 3. Diagnostic value of magnetic resonance imaging in medical meniscus tear.

\begin{tabular}{|l|c|c|}
\hline \multicolumn{1}{|c|}{ Parameter } & Value & 95\% Cl \\
\hline Sensitivity & $61.29 \%$ & $42.19 \%$ to $78.15 \%$ \\
\hline Specificity & $53.85 \%$ & $37.18 \%$ to $69.91 \%$ \\
\hline Positive Likelihood Ratio & 1.33 & 0.86 to 2.06 \\
\hline Negative Likelihood Ratio & 0.72 & 0.42 to 1.22 \\
\hline Accuracy & $57.14 \%$ & - \\
\hline Positive Predictive Value & $51.35 \%$ & $40.48 \%$ to $62.10 \%$ \\
\hline Negative Predictive Value & $63.64 \%$ & $50.75 \%$ to $74.83 \%$ \\
\hline
\end{tabular}

Cl: Confidence interval

had $61.3 \%(42.2 ; 78.2 \%)$ sensitivity, $53.9 \%$ (37.2; 69.9\%) specificity, 51.4\% (40.5; 62.1\%) PPV and 63.6\% (50.8; 74.8\%) NPV in diagnosing LM tear, with $57.1 \%$ accuracy (Table 4).

\section{Medial and Lateral Collateral Ligaments (MCL and LCL) tear}

Among the 70 cases, only one case of MCL tear was diagnosed in arthroscopy, which was also detected in $M R I$ in addition to another false positive. No case of $L C L$ tear was diagnosed.

\section{Discussion}

The knee was the first joint to be examined with arthroscopy in $1919^{[11]}$, while the first MRI scan of the knee was performed in 1985. The current retrospective study was focused on tears of the menisci and cruciate ligaments. In this study, MRI had relatively low sensitivity, especially in meniscus pathology (61.3\% to $92.7 \%)$; and low specificity, especially in cruciate ligaments pathology (33.3\% and 53.9\%). These results are inferior to most of previous studies. In a meta-analysis, which comprised of a review of 29 articles evaluating diagnostic performance of MRI of the menisci and cruciate ligaments, Oei et al. ${ }^{[3]}$ found a pooled weighted sensitivity and specificity of about $93 \%$ and $88 \%$, respectively for medial meniscus tears; about $79 \%$ and $96 \%$ for lateral meniscus tears and about $94 \%$ and $94 \%$ for ACL tear. In this study, an inferior diagnostic performance of MRI may be attributable to a different patient population, different lesions types, pulse sequences used, study design criteria and lack of experienced radiologists to interpret the results. It is believed that the experience level of the radiologist and their expertise is one of the most important factors in maximizing MRI accuracy ${ }^{[8]}$.

The current study demonstrated a higher accuracy of the MRI technique in detecting cruciate ligaments tears than in meniscal tears. Anterior cruciate ligament is one of the most frequently encountered knee ligament injuries. In the United States, there are about $175,000 \mathrm{ACL}$ reconstructions done yearly, while in France $15,000 \mathrm{ACL}$ tears occur per year ${ }^{[12]}$. Therefore, accuracy of the radiological report is a crucial factor in the management of $A C L$ as it impacts the clinical and surgical decision, which conditions knee functional outcomes and may expose to medico-legal issues. In other words, relying on the radiological report for surgery may result in unnecessary procedures in case of false positive imaging finding, which carries additional risks for the knee $^{[13]}$. Considering these risks, orthopedists and surgeons are urged to combine radiological findings with scrupulous clinical examination to improve preoperative diagnostic accuracy and avoid unnecessary arthroscopy. In a 
prospective study carried out using a $0.5 T \mathrm{MRI}$ machine without using a specific imaging protocol, Behairy et al. ${ }^{[14]}$ concluded that the arthroscopy should be done in cases where MRI report does not match with clinical findings or in cases clinically diagnosed as complete ACL tears.

The current study showed a notable difference in the sensitivity and specificity of MRI for detecting medial and lateral meniscal tears. Greater sensitivity was observed in the detection of MM tears, while specificity was relatively higher for LM tears. De Smet et al. ${ }^{[15]}$ found that LM tears are more likely to be missed at MRI diagnosis if the tear involves only one third of the meniscus or is limited to the posterior horn ${ }^{[15]}$. Longitudinal tears of the posterior horn were the most commonly missed tears, as observed retrospectively. The lower sensitivity of MRI in diagnosing LM tears may also be attributable to small tear size, oblique visualization of the posterior horn, the magic angle effect and arterial pulsation artifact ${ }^{[16]}$. In an arthroscopic study of 1086 medial meniscal tears, the posterior horn was involved in $98 \%$ of the torn medial menisci ${ }^{[17]}$. Due to this tear distribution, De Smet ${ }^{[18]}$ believed that radiologists should be cautious in diagnosing a medial meniscus tear that does not involve the posterior horn. On the other hand, Magee and Williams ${ }^{[16]}$ have shown that the sensitivity of diagnosing meniscal tears using 3-Tesla (3-T) fast spin echo MRI with $2 \mathrm{~mm}$-thick cuts was $96 \%$ among a sample of 66 medial and 46 lateral meniscal tears. Hence, using thin slices with a higher signal-to-noise ratio could improve MRI-based diagnosis of meniscal tears.

Diagnostic accuracy of MRI in meniscal tears is comparable between older (50 years old and above) and younger patients when the diagnosis is exclusively based on definite MRI findings ${ }^{[19]}$. However, the equivocal abnormalities involving the free edge of the LM should not be misdiagnosed as tears. This will help prevent unnecessary surgical procedures.

The viewpoint put forth by this study is similar to conclusions drawn from other previous studies. This indicates that, although MRI is a frequently employed presurgical diagnostic method, it is unlikely to replace clinical diagnosis and diagnostic arthroscopy. The use and interpretation of MRI is recommended in conjunction with clinical findings and history to provide a more elaborate and complete preoperative picture of the existing knee pathology.
Improving diagnostic accuracy of MRI in knee pathology would result in inestimable clinical benefits; however, recently improved MRI quality has not consistently enhanced diagnostic precision in meniscal lesions ${ }^{[20]}$. A few measures can be used to improve the overall quality of MRI as a pre-surgical diagnostic technique. Furthermore, MRI scans can be interpreted by 2 different radiologists, which should always be combined with input and feedback obtained from orthopedic surgeons. The diagnostic quality of MRI can also be enhanced by optimizing pulse sequences and using 3-T high field MRI machines.

Limitations of the study include single-center setting, which conditions the findings with the radiological and surgical teams' experience, in addition to MRI scans being interpreted by a single radiologist. Moreover, there was a significant time gap between some MRI examinations and respective arthroscopic procedures.

\section{Conclusion}

Magnetic resonance imaging has inadequate accuracy in preoperative diagnosis of knee pathology including ligament and meniscus injuries. It has moderate sensitivity in diagnosing ligament injuries, especially complete $A C L$ tears and very low specificity in meniscal injury.

In ligament injuries, MRI had higher diagnostic value in posterior ligament injuries including $\mathrm{PCL}$ tears, with $100 \%$ sensitivity and $90.7 \%$ specificity, as compared with $86.7 \%$ and $80.0 \%$ in ACL. In meniscal tear, MRI showed higher sensitivity in detecting medial meniscus tear as compared to tears of the lateral meniscus.

Arthroscopy continues to be the gold standard in the diagnosis of knee pathologies, and preoperative MRI findings should always be interpreted in conjunction with clinical findings and history to provide a more elaborate and complete picture of the knee pathology.

\section{Conflict of Interest}

The authors have no conflict of interest.

\section{Disclosure}

The authors did not receive any type of commercial support either in forms of compensation or financial 
for this study. The author have no financial interest in any of the products or devices, or drugs mentioned in this article.

\section{Ethical Approval}

Obtained.

\section{References}

[1] Dean Deyle G. The role of MRI in musculoskeletal practice: clinical perspective. J Man Manip Ther 2011; 19(3): 152161.

[2] Bridgman S, Richards PJ, Walley G, MacKenzie G, Clement D, McCall I, Griffiths D, Maffulli N. The effect of magnetic resonance imaging scans on knee arthroscopy: randomized controlled trial. Arthroscopy 2007; 23(11): 1167-1173.e1.

[3] Oei EH, Nikken JJ, Verstijnen AC, Ginai AZ, Myriam Hunink MG. MR imaging of the menisci and cruciate ligaments: a systematic review. Radiology 2003; 226(3): 837-848.

[4] MacKenzie R, Lomas DJ, Villar RN, Sands DJ, Teale A, Dixon AK. Magnetic resonance imaging for the problem knee: $A$ randomized clinical trial. Br J Radiol 1993; 66: S21.

[5] Madhusudhan TR, Kumar TM, Bastawrous SS, Sinha A. Clinical examination, MRI and arthroscopy in meniscal and ligamentous knee injuries- a prospective study. J Orthop Surg Res 2008; 3: 19.

[6] Ercin E, Kaya I, Sungur I, Demirbas E, Ugras AA, Cetinus EM. History, clinical findings, magnetic resonance imaging, and arthroscopic correlation in meniscal lesions. Knee Surg Sports Traumatol Arthrosc 2012; 20(5): 851-856.

[7] Hardy JC, Evangelista GT, Grana WA, Huner RE. Accuracy of magnetic resonance imaging of the knee in the community setting. Sports Health 2012; 4(3): 222-231.

[8] Milewski MD, Sanders TG, Miller MD. MRI-arthroscopy correlation: the knee. J Bone Joint Surg An 2011; 93(18): 1735-1745.

[9] Hagino T, Ochiai S, Watanabe $Y$, Senga S, Wako M, Ando T, Sato E, Haro H. Complications after arthroscopic knee surgery. Arch Orthop Trauma Surg 2014; 134(11): 15611564.

[10] Salzler MJ, Lin A, Miller CD, Herold S, Irrgang JJ, Harner CD. Complications after arthroscopic knee surgery. Am J Sports Med 2014; 42(2): 292-296.

[11] Jung JY, Yoon YC, Kwon JW, Ahn JH, Choe BK. Diagnosis of internal derangement of the knee at 3.0-T MR imaging: $3 \mathrm{D}$ isotropic intermediate-weighted versus $2 \mathrm{D}$ sequences. Radiology 2009; 253(3): 780-778.

[12] Guneoun D, Le Corroller T, Amous Z, Pauly V, Sbihi A, Champsaur P. The contribultion of MRI to the diagnosis of traumatic tears of the anterior cruciate ligaments. Diagn Interv Imaging 2012; 93(5): 331-341.
[13] Tsai KJ, Chiang H, Jiang CC. Magnetic resonance imaging of anterior cruciate ligament rupture. BMC Musculoskelet Disord 2004; 5: 21.

[14] Behairy NH, Dorgham MA, Khaled SA. Accuracy of routine magnetic resonance imaging in meniscal and ligamentous injuries of the knee: comparison with arthroscopy. Int Orthop 2009; 33(4): 961-967.

[15] De Smet AA, Mukherjee R. Clinical, MRI, and arthroscopic findings associated with failure to diagnose a lateral meniscal tear on knee MRI. AJR Am J Roentgenol 2008; 190(1): 22-26.

[16] Magee T, Williams D. 3.0-T MRI of meniscal tears. AJR Am J Roentgenol 2006; 187(2): 371375.

[17] Metcalf MH, Barrett GR. Prospective evaluation of 1485 meniscal tear patterns in patients with stable knees. Am J Sports Med 2004; 32(3): 675-680.

[18] De Smet AA. How I diagnose meniscal tears on knee MRI. AJR Am J Roentgenol 2012; 199(3): 481-499.

[19] Subhas N, Sakamoto FA, Mariscalco MW, Polster JM, Obuchowski NA, Jones MH. Accuracy of MRI in the diagnosis of meniscal tears in older patients. AJR Am J Roentgenol 2012; 198(6): W575-580.

[20] Grossman JW, De Smet AA, Shinki K. Comparison of the accuracy rates of 3-T and 1.5-T MRI of the knee in the diagnosis of meniscal tear. AJR Am J Roentgenol 2009; 193(2): 509-514. 


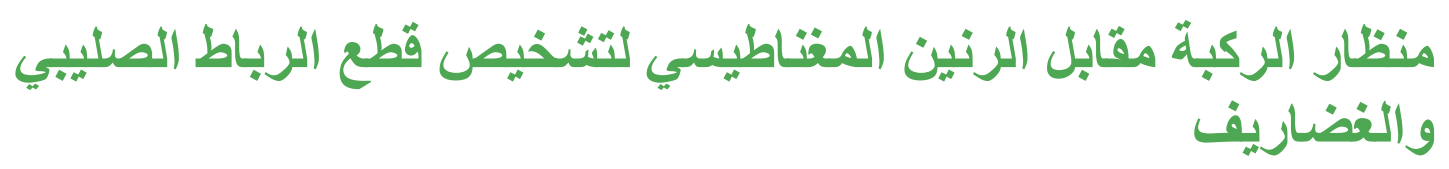

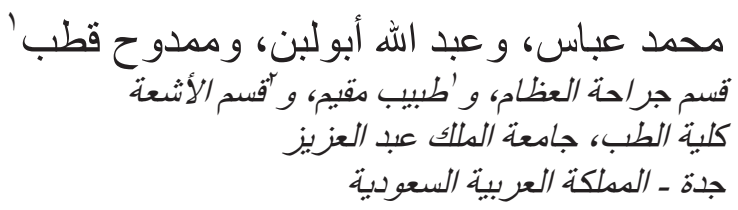

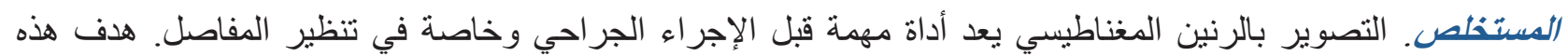

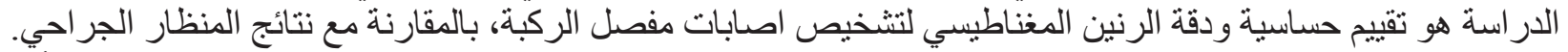

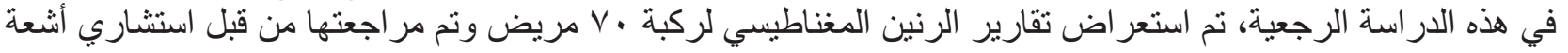

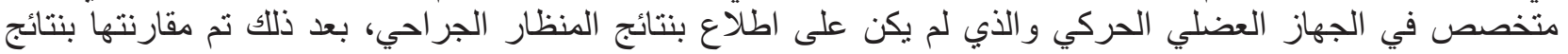

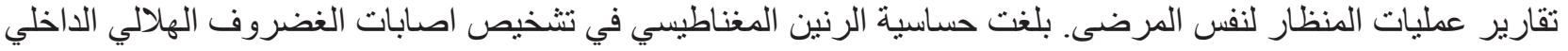

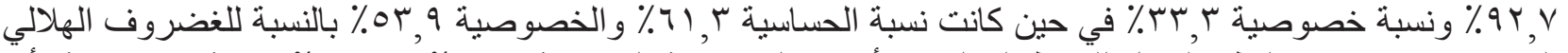

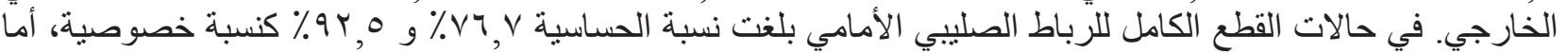

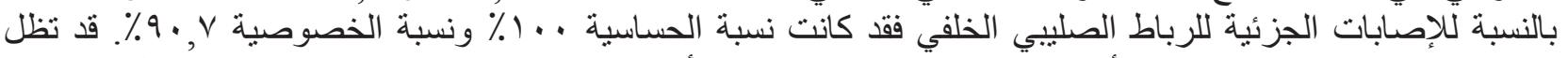
بعض اصابات الرباط الصليبي الأمامي غير مشخصة في تقارير أشعة الرنين المغناطيسي ولذلك بيقى المنظار الوسيلة المنظى لتشخيص اصنيات الربات الركبة. 